\title{
Two-dimensional static deformation of an anisotropic medium
}

\author{
KULDIP SINGH ${ }^{1}$, DINESH KUMAR MADAN ${ }^{2}$, ANITA GOEL $^{3}$ \\ and NAT RAM GARG ${ }^{3}$ \\ ${ }^{1}$ Department of Mathematics, Guru Jambheshwar University, Hisar 125 001, India \\ ${ }^{2}$ Department of Mathematics, T.I.T. \& S., Bhiwani 127 021, India \\ ${ }^{3}$ Department of Mathematics, Maharshi Dayanand University, Rohtak 124 001, \\ India \\ e-mail: nrgmath@rediffmail.com
}

MS received 13 August 2004; revised 28 April 2005

\begin{abstract}
The problem of two-dimensional static deformation of a monoclinic elastic medium has been studied using the eigenvalue method, following a Fourier transform. We have obtained expressions for displacements and stresses for the medium in the transformed domain. As an application of the above theory, the particular case of a normal line-load acting inside an orthotropic elastic half-space has been considered in detail and closed form expressions for the displacements and stresses are obtained. Further, the results for the displacements for a transversely isotropic as well as for an isotropic medium have also been derived in the closed form. The use of matrix notation is straightforward and avoids unwieldy mathematical expressions. To examine the effect of anisotropy, variations of dimensionless displacements for an orthotropic, transversely isotropic and isotropic elastic medium have been compared numerically and it is found that anisotropy affects the deformation significantly.
\end{abstract}

Keywords. Static deformation; anisotropic; orthotropic; monoclinic elastic medium.

\section{Introduction}

Maruyama (1966) obtained closed-form expressions for the displacement and stress fields in a homogeneous isotropic elastic half-space as a result of line-source. Dziewonski \& Anderson (1981) have established that the upper part of the earth is anisotropic. Laminated composite anisotropic materials find a large number of engineering applications. Generalization of the solution to include anisotropy is, however, very difficult. For a transversely isotropic medium, some other relevant contribution are those of Small \& Booker (1984) and Pan (1989).

Chou (1976) and Ting (1995) discussed antiplane strain deformation of an anisotropic medium. Garg et al (1996) obtained representations of seismic sources causing antiplane strain deformations of orthotropic media. The corresponding plane strain deformation of an 
orthotropic elastic medium has been discussed by Garg et al (2003) using an eigenvalue approach. Orthotropic symmetry is exhibited by olivine and orthopyroxens, the principal rock forming minerals of the deep crust and upper mantle.

In the present paper, a novel analytical eigenvalue method is presented for a monoclinic solid. Fourier transformation of the equations of equilibrium for plane strain deformation of a monoclinic solid reduces them into a single linear homogeneous vector differential equation of second order, on which an eigenvalue method is applied to obtain a solution in the transformed domain. The form of the single governing equation for a monoclinic solid derived in this paper is a new contribution to the theory of monoclinic solids. The procedure developed in this paper is relatively simple and straightforward, avoids the cumbersome nature of the problem and is also convenient for numerical computation. As particular cases, normal line-loads acting inside orthotropic, transversely isotropic and perfectly isotropic elastic half-spaces have been considered in detail. The deformation at any point of the medium is useful to analyse the deformation field around mining tremors and drilling into the crust of the earth. It may also find application in various engineering problems, crystal physics and solid-earth geophysics regarding deformation of an anisotropic solid. In fact, the study of a single force acting on a monoclinic solid forms the basis for further investigations such as dipolar sources and faults in an anisotropic solid.

\section{Basic equations and theory}

The equations of equilibrium in the cartesian co-ordinate system $\left(x_{1}, x_{2}, x_{3}\right)$ for zero body forces are

$$
\tau_{i j, j}=0,
$$

where $\tau_{i j}(i, j=1,2,3)$ are the components of the stress tensor.

The stress-strain relations for a monoclinic elastic medium, with $x_{1} x_{2}$-plane as a plane of elastic symmetry are

$$
\begin{aligned}
& \tau_{11}=d_{11} e_{11}+d_{12} e_{22}+d_{13} e_{33}+2 d_{16} e_{12}, \\
& \tau_{22}=d_{12} e_{11}+d_{22} e_{22}+d_{23} e_{33}+2 d_{26} e_{12}, \\
& \tau_{33}=d_{13} e_{11}+d_{23} e_{22}+d_{33} e_{33}+2 d_{36} e_{12}, \\
& \tau_{23}=2 d_{44} e_{23}+2 d_{45} e_{13}, \\
& \tau_{13}=2 d_{45} e_{23}+2 d_{55} e_{13}, \\
& \tau_{12}=d_{16} e_{11}+d_{26} e_{22}+d_{36} e_{33}+2 d_{66} e_{12},
\end{aligned}
$$

where $e_{i j}$ are the components of strain tensor and are related to displacement components $\left(u_{1}, u_{2}, u_{3}\right)$ through the relations

$$
e_{i j}=1 / 2\left(u_{i, j}+u_{j, i}\right) .
$$

The two-suffix symmetric quantities $d_{l k}(l, k=1,2, \ldots, 6)$ are the elastic moduli for a monoclinic elastic medium. For an orthotropic solid with coordinate planes coinciding with the planes of symmetry, we have

$$
d_{16}=d_{26}=d_{36}=d_{45}=0,
$$


and the number of independent elastic coefficients reduce to nine. A transversely isotropic medium is a particular case of orthotropy in which

$$
d_{22}=d_{11}, d_{23}=d_{13}, d_{55}=d_{44}, d_{66}=1 / 2\left(d_{11}-d_{12}\right),
$$

and there are five independent elastic coefficients. Further, isotropy is also a particular case of an orthotropy in which

$$
\begin{aligned}
& d_{11}=d_{22}=d_{33}=\lambda+2 \mu, \\
& d_{12}=d_{13}=d_{23}=\lambda, \\
& d_{44}=d_{55}=d_{66}=\mu,
\end{aligned}
$$

where $\lambda$ and $\mu$ are Lame' constants. For convenience, we shall write

$$
\left(x_{1}, x_{2}, x_{3}\right)=(x, y, z) \quad \text { and } \quad\left(u_{1}, u_{2}, u_{3}\right)=(u, v, w) .
$$

We now consider the plane-strain deformation, parallel to the $x y$-plane, in which the displacement components are independent of $z$ and are of the type

$$
u=u(x, y), v=v(x, y), w=0 .
$$

The non-zero stresses for the plane-strain problem for a monoclinic medium are obtained from (2)-(8), (10) and (12), as

$$
\begin{aligned}
& \tau_{11}=d_{11} \frac{\partial u}{\partial x}+d_{12} \frac{\partial v}{\partial y}+d_{16}\left(\frac{\partial u}{\partial y}+\frac{\partial v}{\partial x}\right), \\
& \tau_{22}=d_{12} \frac{\partial u}{\partial x}+d_{22} \frac{\partial v}{\partial y}+d_{26}\left(\frac{\partial u}{\partial y}+\frac{\partial v}{\partial x}\right), \\
& \tau_{33}=d_{13} \frac{\partial u}{\partial x}+d_{23} \frac{\partial v}{\partial y}+d_{36}\left(\frac{\partial u}{\partial y}+\frac{\partial v}{\partial x}\right), \\
& \tau_{12}=d_{16} \frac{\partial u}{\partial x}+d_{26} \frac{\partial v}{\partial y}+d_{66}\left(\frac{\partial u}{\partial y}+\frac{\partial v}{\partial x}\right) .
\end{aligned}
$$

The equations of equilibrium, for a plane-strain deformation (parallel to the $x y$-plane) of a monoclinic elastic medium are

$$
\begin{aligned}
& {\left[d_{11} \frac{\partial^{2} u}{\partial x^{2}}+d_{66} \frac{\partial^{2} u}{\partial y^{2}}+2 d_{16} \frac{\partial^{2} u}{\partial x \partial y}\right]} \\
& +\left[d_{16} \frac{\partial^{2} v}{\partial x^{2}}+d_{26} \frac{\partial^{2} v}{\partial y^{2}}+\left(d_{12}+d_{66}\right) \frac{\partial^{2} v}{\partial x \partial y}\right]=0, \\
& {\left[d_{16} \frac{\partial^{2} u}{\partial x^{2}}+d_{26} \frac{\partial^{2} u}{\partial y^{2}}+\left(d_{66}+d_{12}\right) \frac{\partial^{2} u}{\partial x \partial y}\right]} \\
& +\left[d_{66} \frac{\partial^{2} v}{\partial x^{2}}+d_{22} \frac{\partial^{2} v}{\partial y^{2}}+2 d_{26} \frac{\partial^{2} v}{\partial x \partial y}\right]=0
\end{aligned}
$$


We define the following dimensionless quantities,

$$
\begin{aligned}
& \alpha=\frac{x}{h}, \beta=\frac{y}{h}, U=\frac{u}{h}, V=\frac{v}{h}, \sigma_{11}=\frac{\tau_{11}}{d_{66}}, \sigma_{12}=\frac{\tau_{12}}{d_{66}}, \sigma_{22}=\frac{\tau_{22}}{d_{66}}, \\
& a=\frac{d_{11}}{d_{66}}, b=\frac{d_{22}}{d_{66}}, c=\frac{d_{12}}{d_{66}}, d=\frac{d_{26}}{d_{66}}, e=\frac{d_{16}}{d_{66}},
\end{aligned}
$$

where ' $h$ ' is a known fixed quantity and has the dimensions of length. Thus, the equations of equilibrium (17) and (18), in dimensionless form become

$$
\begin{aligned}
& {\left[a \frac{\partial^{2} U}{\partial \alpha^{2}}+\frac{\partial^{2} U}{\partial \beta^{2}}+2 e \frac{\partial^{2} U}{\partial \alpha \partial \beta}\right]+\left[e \frac{\partial^{2} V}{\partial \alpha^{2}}+d \frac{\partial^{2} V}{\partial \beta^{2}}+(c+1) \frac{\partial^{2} V}{\partial \alpha \partial \beta}\right]=0,} \\
& {\left[e \frac{\partial^{2} U}{\partial \alpha^{2}}+d \frac{\partial^{2} U}{\partial \beta^{2}}+(1+c) \frac{\partial^{2} U}{\partial \alpha \partial \beta}\right]+\left[\frac{\partial^{2} V}{\partial \alpha^{2}}+b \frac{\partial^{2} V}{\partial \beta^{2}}+2 d \frac{\partial^{2} V}{\partial \alpha \partial \beta}\right]=0 .}
\end{aligned}
$$

In (20) and (21), $\alpha$ is the dimensionless vertical distance, $\beta$ is the dimensionless horizontal distance, $U$ is the dimensionless vertical displacement, $V$ is the dimensionless horizontal displacement, $\sigma_{11}, \sigma_{12}, \sigma_{22}$ are the dimensionless stress components, and $a, b, c, d, e$ are dimensionless elastic coefficients.

For an isotropic solid,

$$
a=b=1 /\left(1-\alpha^{*}\right), c=\left(1-2 \alpha^{*}\right) /\left(\alpha^{*}-1\right)=a-2, d=0, e=0,
$$

where

$$
\alpha^{*}=(\lambda+\mu) /(\lambda+2 \mu),
$$

in particular, for a Poissonian isotropic solid $(\lambda=\mu)$, and $\alpha^{*}=2 / 3$.

We define the Fourier transform $\bar{f}(\alpha, k)$ of a function $f(\alpha, \beta)$ by the relation

$$
\bar{f}(\alpha, k)=F[f(\alpha, \beta)]=\int_{-\infty}^{+\infty} f(\alpha, \beta) e^{\imath k \beta} \mathrm{d} \beta,
$$

so that

$$
f(\alpha, \beta)=(1 / 2 \pi) \int_{-\infty}^{\infty} \bar{f}(\alpha, k) e^{-\imath k \beta} \mathrm{d} k,
$$

where $k$ is the Fourier transform parameter.

Applying the Fourier transformation on equations of equilibrium (20) and (21), we find

$$
\begin{aligned}
& {\left[a \frac{\mathrm{d}^{2} \bar{U}}{\mathrm{~d} \alpha^{2}}-2 e \iota k \frac{\mathrm{d} \bar{U}}{\mathrm{~d} \alpha}-k^{2} \bar{U}\right]+\left[e \frac{\mathrm{d}^{2} \bar{V}}{\mathrm{~d} \alpha^{2}}-\iota k(1+c) \frac{\mathrm{d} \bar{V}}{\mathrm{~d} \alpha}-\mathrm{d} k^{2} \bar{V}\right]=0,} \\
& {\left[e \frac{\mathrm{d}^{2} \bar{U}}{\mathrm{~d} \alpha^{2}}-\iota k(1+c) \frac{\mathrm{d} \bar{U}}{\mathrm{~d} \alpha}-k^{2} d \bar{U}\right]+\left[\frac{\mathrm{d}^{2} \bar{V}}{\mathrm{~d} \alpha^{2}}-2 d \iota k \frac{\mathrm{d} \bar{V}}{\mathrm{~d} \alpha}-b k^{2} \bar{V}\right]=0 .}
\end{aligned}
$$

The above two equations can be written as the following single homogeneous vector differential equation:

$$
A^{\circ} \frac{\mathrm{d}^{2} N_{0}}{\mathrm{~d} \alpha^{2}}+B^{\circ} \frac{\mathrm{d} N_{0}}{\mathrm{~d} \alpha}+C^{\circ} N_{0}=0,
$$


where

$$
\begin{aligned}
A^{\circ} & =\left[\begin{array}{ll}
a & e \\
e & 1
\end{array}\right], B^{\circ}=\left[\begin{array}{cc}
-2 \iota k e & -\imath k(1+c) \\
-\imath k(1+c) & -2 \imath k d
\end{array}\right], \\
C^{\circ} & =\left[\begin{array}{cc}
-k^{2} & -k^{2} d \\
-k^{2} d & -b k^{2}
\end{array}\right], N_{0}=\left[\frac{\bar{U}}{V}\right] .
\end{aligned}
$$

We note that matrices $A^{\circ}, B^{\circ}$ and $C^{\circ}$ are all symmetric, matrix $A^{\circ}$ depends upon dimensionless elastic moduli while the matrices $B^{\circ}$ and $C^{\circ}$ depend upon parameter $k$ also. The governing equation (28) for a monoclinic solid is a new contribution and eigenvalue method shall be applied to solve the problem. For this, we seek a solution of vector differential equation (28) of the type

$$
N_{0}(\alpha, k)=E(k) e^{s \alpha},
$$

where $s$ is a parameter and $E(k)$ is a matrix of the type $2 \times 1$. Substitution of the value of $N_{0}$ from (30) into vector differential equation (28) gives the following characteristic equation:

$$
a_{0} s^{4}+4 a_{1} s^{3}+6 a_{2} s^{2}+4 a_{3} s+a_{4}=0,
$$

where

$$
\begin{aligned}
& a_{0}=a-e^{2}, \quad a_{1}=-\iota(a d-c e) k / 2, \\
& a_{2}=\frac{-\left(a b-c^{2}-2 c+2 e d\right) k^{2}}{6}, \\
& a_{3}=\frac{2 \iota(c d-b e) k^{3}}{4}, \quad a_{4}=\left(b-d^{2}\right) k^{4} .
\end{aligned}
$$

To solve biquadratic equation (31), we make use of Descartes' method, in which the second term is removed by using the transformation

$$
z^{*}=a_{0} s+a_{1},
$$

so that (31) reduces to

$$
\left(z^{*}\right)^{4}+6 H\left(z^{*}\right)^{2}+4 G z^{*}+\left(a_{0}^{2} I-3 H^{2}\right)=0,
$$

where

$$
\begin{aligned}
H & =a_{0} a_{2}-a_{1}^{2}, G=a_{0}^{2} a_{3}-3 a_{0} a_{1} a_{2}+2 a_{1}^{3}, \\
I & =a_{0} a_{4}-4 a_{1} a_{3}+3 a_{2}^{2} .
\end{aligned}
$$

Following Descartes' method, the left hand side of (34) can be resolved into a product of two quadratic factors as

$$
\begin{aligned}
& \left(z^{*}\right)^{4}+6 H\left(z^{*}\right)^{2}+4 G z^{*}+\left(a_{0}^{2} I-3 H^{2}\right) \\
& \quad=\left[\left(z^{*}\right)^{2}+p_{0} z^{*}+q\right]\left[\left(z^{*}\right)^{2}-p_{0} z^{*}+q^{\prime}\right],
\end{aligned}
$$


where

$$
\begin{aligned}
& q+q^{\prime}=p_{0}^{2}+6 H, q q^{\prime}=a_{0}^{2} I-3 H^{2}, \\
& p_{0}\left(q^{\prime}-q\right)=4 G .
\end{aligned}
$$

when $p_{0}=0$, the values of $q$ and $q$ ' are determined from (37) directly, otherwise, eliminating $q$ and $q$ ' from (37) and (38), we get

$$
\xi^{3}+3 b_{1} \xi^{2}+3 b_{2} \xi+b_{3}=0
$$

where

$$
\begin{aligned}
\xi & =p_{0}^{2}, \quad b_{1}=4 H, \\
b_{2} & =4\left(12 H^{2}-a_{0}^{2} I\right) / 3, b_{3}=-16 G^{2} .
\end{aligned}
$$

Equation (39) is a cubic in $\xi$ and hence, can be solved using Cardan's method and thus (39) is reduced to

$$
\delta^{3}+3 H_{1} \delta+G_{1}=0
$$

where

$$
\delta=\xi+b_{1}, H_{1}=b_{2}-b_{1}^{2}, G_{1}=b_{3}-3 b_{1} b_{2}+2 b_{1}^{3} .
$$

The roots of the cubic equation (41) are

$$
\delta=u_{0}+v_{0},
$$

where $u_{0}$ and $v_{0}$ are given by

$$
u_{0}^{3}=\frac{-G_{1}+\sqrt{G_{1}^{2}+4 H_{1}^{3}}}{2}, v_{0}^{3}=\frac{-G_{1}-\sqrt{G_{1}^{2}+4 H_{1}^{3}}}{2},
$$

satisfying $u_{0} v_{0}=-H_{1}$. Then $p_{0}$ is given by

$$
p_{0}^{2}=\delta-b_{1},
$$

and $q$ and $q^{\prime}$ are then to be calculated from (37), (38) and (45). Equation (36) gives

$$
\begin{aligned}
& z_{1}^{*}=\frac{-p_{0}+\left(p_{0}^{2}-4 q\right)^{1 / 2}}{2}, z_{2}^{*}=\frac{-p_{0}-\left(p_{0}^{2}-4 q\right)^{1 / 2}}{2}, \\
& z_{3}^{*}=\frac{p_{0}+\left(p_{0}^{2}-4 q^{\prime}\right)^{1 / 2}}{2}, z_{4}^{*}=\frac{p_{0}-\left(p_{0}^{2}-4 q^{\prime}\right)^{1 / 2}}{2}
\end{aligned}
$$

as the roots of the biquadratic equation (34). Hence, the eigenvalues of the problem are $s_{1}, s_{2}, s_{3}, s_{4}$, where

$$
s_{L}=\frac{z_{L}^{*}-a_{1}}{a_{0}}, \text { for } L=1,2,3,4
$$


The eigenvectors for a monoclinic elastic medium are obtained by solving the matrix equation

$$
\left[s^{2} A^{\circ}+s B^{\circ}+C^{\circ}\right] E(k)=0,
$$

and the eigenvectors are found to be

$$
X_{L}^{T}=\left[P_{1 L}, 1\right], \text { for } L=1,2,3,4,
$$

where

$$
P_{1 L}=\frac{-\left[e s_{L}^{2}-\imath k s_{L}(1+c)-k^{2} d\right]}{a s_{L}^{2}-2 \iota k e s_{L}-k^{2}}=\frac{-\left[s_{L}^{2}-2 \iota k d s_{L}-b k^{2}\right]}{e s_{L}^{2}-\iota k s_{L}(1+c)-k^{2} d}
$$

and $(\ldots)^{T}$ denote the transpose of the matrix $(\ldots)$. Thus, a general solution of the vectormatrix differential equation (28) for a monoclinic elastic medium for non-repeated eigenvalues is of the form

$$
N_{0}(\alpha, k)=\sum_{L=1}^{4}\left(B_{L} X_{L} e^{s_{L} \alpha}\right),
$$

where coefficients $B_{1}, B_{2}, B_{3}$ and $B_{4}$ are to be determined from prescribed boundary conditions. These coefficients may depend upon $k$. Equation (51) gives the following expressions for the dimensionless displacements in the transformed domain.

$$
\begin{aligned}
& \bar{U}(\alpha, k)=B_{1} P_{11} e^{s_{1} \alpha}+B_{2} P_{12} e^{s_{2} \alpha}+B_{3} P_{13} e^{s_{3} \alpha}+B_{4} P_{14} e^{s_{4} \alpha}, \\
& \bar{V}(\alpha, k)=B_{1} e^{s_{1} \alpha}+B_{2} e^{s_{2} \alpha}+B_{3} e^{s_{3} \alpha}+B_{4} e^{s_{4} \alpha} .
\end{aligned}
$$

Further, the expressions for dimensionless stresses for a plane strain deformation for a monoclinic solid are found to be

$$
\begin{aligned}
& \bar{\sigma}_{11}=\left[Q_{11} B_{1} e^{s_{1} \alpha}+Q_{12} B_{2} e^{s_{2} \alpha}+Q_{13} B_{3} e^{s_{3} \alpha}+Q_{14} B_{4} e^{s_{4} \alpha}\right], \\
& \bar{\sigma}_{12}=\left[R_{11} B_{1} e^{s_{1} \alpha}+R_{12} B_{2} e^{s_{2} \alpha}+R_{13} B_{3} e^{s_{3} \alpha}+R_{14} B_{4} e^{s_{4} \alpha}\right],
\end{aligned}
$$

where

$$
\begin{aligned}
& Q_{1 L}=a P_{1 L} s_{L}-\imath k c-\imath k e P_{1 L}+s_{L} e, \\
& R_{1 L}=e P_{1 L} s_{L}-\imath k d-\imath k P_{1 L}+s_{L} \text { for } L=1,2,3,4 .
\end{aligned}
$$

\section{Particular cases}

\subsection{Orthotropic symmetry}

In this case

$$
d=0, e=0, a_{1}=0, a_{3}=0,
$$

and vector differential equation and characteristics equation for an orthotropic medium are obtained from (28), (29), (31), (32) and (55). We find

$$
A \frac{\mathrm{d}^{2} N}{\mathrm{~d} \alpha^{2}}+B \frac{\mathrm{d} N}{\mathrm{~d} \alpha}+C N=0
$$


in which $\mathrm{A}, \mathrm{B}, \mathrm{C}$ are

$$
\begin{aligned}
& A=\left(\begin{array}{ll}
a & 0 \\
0 & 1
\end{array}\right), \quad B=\left(\begin{array}{cc}
0 & -\imath k(1+c) \\
-\imath k(1+c) & 0
\end{array}\right), \\
& C=\left(\begin{array}{cc}
-k^{2} & 0 \\
0 & -b k^{2}
\end{array}\right), N=\left(\begin{array}{c}
\bar{U} \\
\bar{V}
\end{array}\right),
\end{aligned}
$$

and

$$
a s^{4}-\left(a b-c^{2}-2 c\right) k^{2} s^{2}+b k^{4}=0 .
$$

The characteristic equation (58) is a quadratic equation in $\mathrm{s}^{2}$ and can be solved directly without using Descartes' method and gives the eigenvalues as

$$
s^{2}=m_{1}^{2} k^{2}, m_{2}^{2} k^{2},
$$

where

$$
\begin{aligned}
& m_{1}^{2}=\frac{A_{0}+\left(A_{0}^{2}-4 B_{0}\right)^{1 / 2}}{2}, \quad m_{2}^{2}=\frac{A_{0}-\left(A_{0}^{2}-4 B_{0}\right)^{1 / 2}}{2}, \\
& A_{0}=\left(a b-c^{2}-2 c\right) / a=m_{1}^{2}+m_{2}^{2}, B_{0}=b / a=m_{1}^{2} m_{2}^{2} .
\end{aligned}
$$

Under the assumption that $m_{1} \neq m_{2}$, the eigenvalues are

$$
\begin{aligned}
& s_{1}=m_{1}|k|, s_{2}=m_{2}|k|, \\
& s_{3}=-m_{1}|k|, s_{4}=-m_{2}|k|,
\end{aligned}
$$

with real parts of $\left(m_{1}, m_{2}\right)$ as positive.

The eigenvectors for an orthotropic elastic medium are given by

$$
X_{L}^{T}=\left[P_{2 L}, 1\right], X_{L+2}^{T}=\left[-P_{2 L}, 1\right],
$$

for $L=1,2$, in which

$$
\begin{aligned}
& P_{21}=\frac{\iota m_{1}|k|}{k}\left(\frac{1+c}{a m_{1}^{2}-1}\right)=\frac{\iota k}{m_{1}|k|}\left(\frac{b-m_{1}^{2}}{1+c}\right), \\
& P_{22}=\frac{\iota m_{2}|k|}{k}\left(\frac{1+c}{a m_{2}^{2}-1}\right)=\frac{\iota k}{m_{2}|k|}\left(\frac{b-m_{2}^{2}}{1+c}\right),
\end{aligned}
$$

Thus, a solution of matrix equation (56) for the case of an orthotropic elastic medium is

$$
N(\alpha, k)=\sum_{L=1}^{2}\left[\left(C_{L} X_{L} e^{m_{L}|k| \alpha}+C_{L+2} X_{L+2} e^{-m_{L}|k| \alpha}\right],\right.
$$

where the coefficients $C_{1}, C_{2}, C_{3}$ and $C_{4}$ are to be determined from boundary conditions and they may depend upon $k$. 
The transformed dimensionless displacements for an orthotropic elastic medium are

$$
\begin{aligned}
& \bar{U}(\alpha, k)=C_{1} P_{21} e^{m_{1}|k| \alpha}+C_{2} P_{22} e^{m_{2}|k| \alpha}-C_{3} P_{21} e^{-m_{1}|k| \alpha}-C_{4} P_{22} e^{-m_{2}|k| \alpha}, \\
& \bar{V}(\alpha, k)=C_{1} e^{m_{1}|k| \alpha}+C_{2} e^{m_{2}|k| \alpha}+C_{3} e^{-m_{1}|k| \alpha}+C_{4} e^{-m_{2}|k| \alpha},
\end{aligned}
$$

and stresses in the transformed domain are

$$
\begin{aligned}
& \bar{\sigma}_{11}(\alpha, k)=Q_{21} C_{1} e^{m_{1}|k| \alpha}+Q_{22} C_{2} e^{m_{2}|k| \alpha}+Q_{21} C_{3} e^{-m_{1}|k| \alpha}+Q_{22} C_{4} e^{-m_{2}|k| \alpha}, \\
& \bar{\sigma}_{12}(\alpha, k)=R_{21} C_{1} e^{m_{1}|k| \alpha}+R_{22} C_{2} e^{m_{2}|k| \alpha}-R_{21} C_{3} e^{-m_{1}|k| \alpha}-R_{22} C_{4} e^{-m_{2}|k| \alpha},
\end{aligned}
$$

where

$$
\begin{aligned}
& Q_{2 L}=a P_{2 L} m_{L}|k|-\imath c k, \\
& R_{2 L}=m_{L}|k|-\imath P_{2 L} k, \text { for } L=1,2 .
\end{aligned}
$$

\subsection{Transversely isotropic symmetry}

This case is a particular case of monoclinic symmetry as well as orthotropic symmetry and here the dimensionless elastic constants are

$$
a=b=2\left(\frac{d_{11}}{d_{11}-d_{12}}\right), c=2\left(\frac{d_{12}}{d_{11}-d_{12}}\right)=a-2, d=e=0 .
$$

The characteristic equation for a transversely isotropic elastic medium becomes

$$
s^{4}-2 k^{2} s^{2}+k^{4}=0
$$

provided $d_{11} \neq 0$ and $d_{11} \neq d_{12}$ and it is observed that this characteristic equation is independent of its elastic moduli $a, b, c$. The solution of characteristic equation (69) gives repeated eigenvalues

$$
s=s_{1}=s_{2}=-s_{3}=-s_{4}=|k| .
$$

The equilibrium equations in the transformed domain for a transversely isotropic medium are equivalent to the first-order vector differential equation. Further treatment for this case is different from the treatment discussed earlier for a orthotropic/monoclinic medium with different eigenvalues.

Ross (1984) has given a procedure to tackle the problems with repeated eigenvalues provided the governing vector differential equation is of the first-order. For this situation, we define the process as given by Ross (1984) and the first order vector differential equation representing the equilibrium equations is

$$
\mathrm{d} N_{1} / \mathrm{d} \alpha=A_{1} N_{1},
$$

where

$$
N_{1}=\left[\begin{array}{c}
\bar{U} \\
\bar{V} \\
\frac{d \bar{U}}{d \alpha} \\
\frac{d \bar{V}}{d \alpha}
\end{array}\right], A_{1}=\left[\begin{array}{cccc}
0 & 0 & 1 & 0 \\
0 & 0 & 0 & 1 \\
\frac{k^{2}}{a} & 0 & 0 & \frac{\iota k(a-1)}{a} \\
0 & a k^{2} & \iota k(a-1) & 0
\end{array}\right]
$$


Following the procedure as outlined by Ross (1984), the four independent eigen vectors are found to be

$$
\begin{aligned}
& X_{1}=\left(\begin{array}{c}
\iota|k| \\
k \\
\iota k^{2} \\
k|k|
\end{array}\right), X_{2}=\left[\begin{array}{c}
\iota\left\{|k| \alpha-\left(\frac{2 a}{a-1}\right)\right\} \\
k\left(\alpha-\frac{1}{|k|}\right) \\
\iota|k|\left\{|k| \alpha-\left(\frac{1+a}{a-1}\right)\right\} \\
k|k| \alpha
\end{array}\right], \\
& X_{3}=\left(\begin{array}{c}
-\iota|k| \\
k \\
\iota k^{2} \\
-k|k|
\end{array}\right), X_{4}=\left[\begin{array}{c}
-\iota\left\{k \mid \alpha+\left(\frac{2 a}{a-1}\right)\right\} \\
k\left(\alpha+\frac{1}{|k|}\right) \\
\iota|k|\left\{|k| \alpha+\left(\frac{1+a}{a-1}\right)\right\} \\
-k|k| \alpha
\end{array}\right)
\end{aligned}
$$

Thus, a general solution of first order matrix differential equation (71) for a transversely isotropic medium (Ross 1984) is

$$
N_{1}=\left(D_{1} X_{1}+D_{2} X_{2}\right) e^{|k| \alpha}+\left(D_{3} X_{3}+D_{4} X_{4}\right) e^{-|k| \alpha},
$$

where $D_{1}, D_{2}, D_{3}, D_{4}$ are coefficients which may depend upon $k$ and dimensionless elastic coefficient $a$.

The displacements and stresses in the transformed domain for a transversely isotropic medium are found to be

$$
\begin{aligned}
\bar{U}= & \iota\left[\left\{D_{1}|k|+D_{2}\left(|k| \alpha-\frac{2 a}{a-1}\right)\right\} e^{|k| \alpha}\right. \\
& \left.-\left\{D_{3}|k|+D_{4}\left(|k| \alpha+\frac{2 a}{a-1}\right)\right\} e^{-|k| \alpha}\right] \\
\bar{V}= & k\left[\left\{D_{1}+D_{2}\left(\alpha-\frac{1}{|k|}\right)\right\} e^{|k| \alpha}+\left\{D_{3}+D_{4}\left(\alpha+\frac{1}{|k|}\right)\right\} e^{-|k| \alpha}\right] \\
\bar{\sigma}_{11}= & \iota\left[2 k^{2} D_{1} e^{|k| \alpha}+\left\{2 k^{2} \alpha+|k|\left(\frac{2-4 a}{a-1}\right)\right\} D_{2} e^{|k| \alpha}\right. \\
& \left.+2 k^{2} D_{3} e^{-|k| \alpha}+\left\{2 k^{2} \alpha+|k|\left(\frac{4 a-2}{a-1}\right)\right\} D_{4} e^{-|k| \alpha}\right], \\
\bar{\sigma}_{12}= & {\left[\left\{2 k|k| D_{1}+D_{2}\left(2 k|k| \alpha-\frac{2 a k}{a-1}\right)\right\} e^{|k| \alpha}\right.} \\
& \left.-\left\{2 k|k| D_{3}+D_{4}\left(2 k|k| \alpha+\frac{2 a k}{a-1}\right)\right\} e^{-|k| \alpha}\right] .
\end{aligned}
$$




\section{An application: A normal line-load in an elastic half-space}

We consider an elastic half-space with $x$-axis vertically downwards and the origin of the cartesian coordinate system $(x, y, z)$ is taken at the boundary of the half-space. Let a normal line-load $P_{0}$ be acting vertically downwards on a line parallel to $z$-axis and passing through the point $(H, 0)$. The bounding surface $x=0$ is assumed to be stress-free and we shall calculate the resulting stresses and displacements at any point of the half-space for the following three types of medium:

\subsection{Deformation of an orthotropic elastic medium}

Consider the elastic half-space consisting of region $\alpha<\alpha_{0}$ (medium I) and region $\alpha>\alpha_{0}$ (medium II). We find the displacements and stresses at any point of an orthotropic elastic halfspace due to a normal line-load $P_{0}$ acting vertically downwards on a line parallel to $z$-axis (figure 1).

The boundary conditions are:

On the plane $x=0$,

$$
\tau_{11}^{1}(x=0, y)=0, \tau_{12}^{1}(x=0, y)=0 .
$$

On the plane $x=H$,

$$
\begin{aligned}
& u^{1}(x=H, y)=u^{2}(x=H, y), \\
& v^{1}(x=H, y)=v^{2}(x=H, y), \\
& \tau_{12}^{1}(x=H, y)=\tau_{12}^{2}(x=H, y), \\
& \tau_{11}^{2}(x=H, y)-\tau_{11}^{1}(x=H, y)=-P_{0} \delta(y),
\end{aligned}
$$

where $\delta(y)$ is the Dirac-delta function and $F[\delta(y)]=1$.

Defining the dimensionless force

$$
P=P_{0} / d_{66}
$$

Let the coefficients $C_{1}, C_{2}, C_{3}, C_{4}$ appearing in (65) and (66) in medium I be represented by $\bar{C}_{1}, \bar{C}_{2}, \bar{C}_{3}, \bar{C}_{4}$ and for medium II be represented by $C_{1}^{+}, C_{2}^{+}, C_{3}^{+}, C_{4}^{+}$.

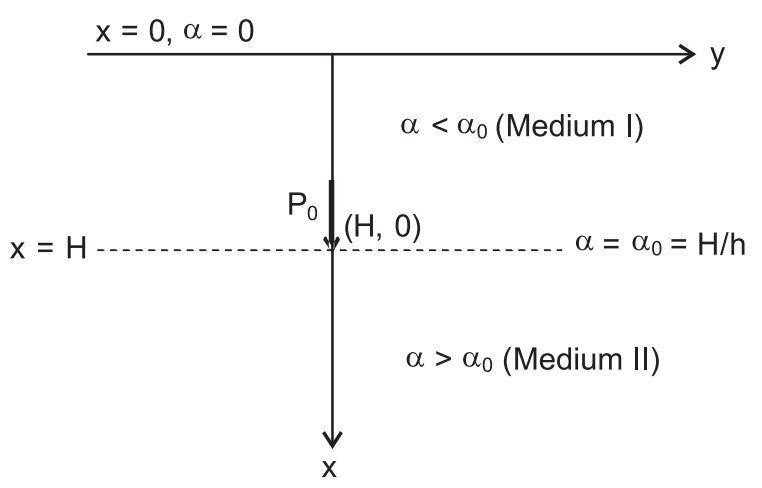

Figure 1. A normal line-load $P_{0}$. 
The corresponding response in medium I is

$$
\begin{aligned}
\bar{U}^{1}(\alpha, k)= & \bar{C}_{1} P_{21} e^{m_{1}|k| \alpha}+\bar{C}_{2} P_{22} e^{m_{2}|k| \alpha}-\bar{C}_{3} P_{21} e^{-m_{1}|k| \alpha}-\bar{C}_{4} P_{22} e^{-m_{2}|k| \alpha} \\
\bar{V}^{1}(\alpha, k)= & \bar{C}_{1} e^{m_{1}|k| \alpha}+\bar{C}_{2} e^{m_{2}|k| \alpha}+\bar{C}_{3} e^{-m_{1}|k| \alpha}+\bar{C}_{4} e^{-m_{2}|k| \alpha} \\
\bar{\sigma}_{11}^{1}(\alpha, k)= & \left(a P_{21} m_{1}|k|-\iota k c\right)\left(\bar{C}_{1} e^{m_{1}|k| \alpha}+\bar{C}_{3} e^{-m_{1}|k| \alpha}\right) \\
& +\left(a P_{22} m_{2}|k|-\iota k c\right)\left(\bar{C}_{2} e^{m_{2}|k| \alpha}+\bar{C}_{4} e^{-m_{2}|k| \alpha}\right) \\
\bar{\sigma}_{12}^{1}(\alpha, k)= & \left(m_{1}|k|-\iota k P_{21}\right)\left(\bar{C}_{1} e^{m_{1}|k| \alpha}-\bar{C}_{3} e^{-m_{1}|k| \alpha}\right) \\
& +\left(m_{2}|k|-\iota k P_{22}\right)\left(\bar{C}_{2} e^{m_{2}|k| \alpha}-\bar{C}_{4} e^{-m_{2}|k| \alpha}\right),
\end{aligned}
$$

and in medium II is given by,

$$
\begin{aligned}
& \bar{U}^{2}(\alpha, k)=-C_{3}^{+} P_{21} e^{-m_{1}|k| \alpha}-C_{4}^{+} P_{22} e^{-m_{2}|k| \alpha}, \\
& \bar{V}^{2}(\alpha, k)=C_{3}^{+} e^{-m_{1}|k| \alpha}+C_{4}^{+} e^{-m_{2}|k| \alpha} \\
& \bar{\sigma}_{11}^{2}(\alpha, k)=\left(a P_{21} m_{1}|k|-\imath k c\right)\left(C_{3}^{+} e^{-m_{1}|k| \alpha}\right)+\left(a P_{22} m_{2}|k|-\iota k c\right)\left(C_{4}^{+} e^{-m_{2}|k| \alpha}\right), \\
& \bar{\sigma}_{12}^{2}(\alpha, k)=\left(m_{1}|k|-\iota k P_{21}\right) \bar{C}_{3} e^{-m_{1}|k| \alpha}\left(m_{2}|k|-\imath k P_{22}\right) \bar{C}_{4} e^{-m_{2}|k| \alpha} .
\end{aligned}
$$

Applying the boundary conditions from (77)-(78) and using the matrix method, we obtain the following values of coefficients.

$$
\begin{aligned}
\bar{C}_{1} & =M_{3} / D_{5}, \bar{C}_{2}=-M_{3} / D_{6}, \\
\bar{C}_{3} & =\frac{-M_{3}}{M_{1}}\left(\frac{M_{2}}{D_{5}}-\frac{2 D_{2} D_{4}}{D_{6}}\right), \bar{C}_{4}=\frac{-M_{3}}{M_{1}}\left(\frac{M_{2}}{D_{6}}-\frac{2 D_{1} D_{3}}{D_{5}}\right), \\
C_{3}^{+} & =\frac{-M_{3}}{D_{7}}\left[1+\frac{M_{2}}{M_{1}} \frac{D_{7}}{D_{5}}-\frac{2}{M_{1}} \frac{D_{2} D_{4} D_{7}}{D_{6}}\right], \\
C_{4}^{+} & =\frac{M_{3}}{D_{8}}\left[1-\frac{M_{2}}{M_{1}} \frac{D_{8}}{D_{6}}+\frac{2}{M_{1}} \frac{D_{1} D_{3} D_{8}}{D_{5}}\right],
\end{aligned}
$$

where

$$
\begin{array}{ll}
D_{1}=a P_{21} m_{1}|k|-\imath k c, & D_{2}=a P_{22} m_{2}|k|-\imath k c, \\
D_{3}=m_{1}|k|-\iota k P_{21}, & D_{4}=m_{2}|k|-\imath k P_{22}, \\
D_{5}=e^{m_{1}|k| \alpha_{0}}, & D_{6}=e^{m_{2}|k| \alpha_{0}}, \\
D_{7}=e^{-m_{1}|k| \alpha_{0}}, & D_{8}=e^{-m_{2}|k| \alpha_{0}} . \\
M_{1}=D_{1} D_{4}-D_{2} D_{3}, & M_{2}=D_{1} D_{4}+D_{2} D_{3}, \\
M_{3}=P / 2\left(D_{1}-D_{2}\right) . &
\end{array}
$$


Putting values of the various coefficients from (82) into displacements and stresses obtained in (80)-(81), we get the following expressions for displacements and stresses at any point of an orthotropic elastic half-space in the transformed domain:

$$
\begin{aligned}
& \bar{U}(\alpha, k)=\frac{P}{2 T_{1}}\left[m_{1} R_{1} \frac{1}{|k|} e^{-m_{1}|k|\left|\alpha-\alpha_{0}\right|}-m_{2} R_{2} \frac{1}{|k|} e^{-m_{2}|k|\left|\alpha-\alpha_{0}\right|}\right. \\
& +\frac{m_{1} R_{1} T_{3}}{T_{2}} \frac{1}{|k|} e^{-m_{1}|k|\left(\alpha+\alpha_{0}\right)}+\frac{m_{2} R_{2} T_{3}}{T_{2}} \frac{1}{|k|} e^{-m_{2}|k|\left(\alpha+\alpha_{0}\right)} \\
& \left.+\frac{2 m_{1} R_{1} T_{4}}{T_{2}} \frac{1}{|k|} e^{-|k|\left(m_{1} \alpha+m_{2} \alpha_{0}\right)}-\frac{2 m_{2} R_{2} T_{5}}{T_{2}} \frac{1}{|k|} e^{-|k|\left(m_{2} \alpha+m_{1} \alpha_{0}\right)}\right] \\
& \bar{V}(\alpha, k)=\frac{P}{2 \iota T_{1}}\left[ \pm \frac{1}{k} e^{-m_{1}|k|\left|\alpha-\alpha_{0}\right|} \mp \frac{1}{k} e^{-m_{2}|k|\left|\alpha-\alpha_{0}\right|}\right. \\
& -\frac{T_{3}}{T_{2}} \frac{1}{k} e^{-m_{1}|k|\left(\alpha+\alpha_{0}\right)}-\frac{T_{3}}{T_{2}} \frac{1}{k} e^{-m_{2}|k|\left(\alpha+\alpha_{0}\right)} \\
& \left.+\frac{2 T_{4}}{T_{2}} \frac{1}{k} e^{-|k|\left(m_{1} \alpha+m_{2} \alpha_{0}\right)}+\frac{2 T_{5}}{T_{2}} \frac{1}{k} e^{-|k|\left(m_{1} \alpha_{0}+m_{2} \alpha\right)}\right], \\
& \bar{\sigma}_{11}=\frac{P}{2 T_{1}}\left[ \pm\left(a m_{1}^{2} R_{1}-c\right) e^{-m_{1}|k|\left|\alpha-\alpha_{0}\right|} \mp\left(a m_{2}^{2} R_{2}-c\right) e^{-m_{2}|k|\left|\alpha-\alpha_{0}\right|}\right. \\
& -\frac{T_{3}}{T_{2}}\left(a m_{1}^{2} R_{1}-c\right) e^{-m_{1}|k|\left(\alpha+\alpha_{0}\right)}-\frac{T_{3}}{T_{2}}\left(a m_{2}^{2} R_{2}-c\right) e^{-m_{2}|k|\left(\alpha+\alpha_{0}\right)} \\
& +\frac{2 T_{4}}{T_{2}}\left(a m_{1}^{2} R_{1}-c\right) e^{-|k|\left(m_{1} \alpha+m_{2} \alpha_{0}\right)} \\
& \left.+\frac{2 T_{5}}{T_{2}}\left(a m_{2}^{2} R_{2}-c\right) e^{-|k|\left(m_{2} \alpha+m_{1} \alpha_{0}\right)}\right], \\
& \bar{\sigma}_{12}=\frac{\iota P|k|}{T_{1} k}\left[\frac{-m_{1}}{2}\left(1+R_{1}\right) e^{-m_{1}|k|\left|\alpha-\alpha_{0}\right|}+\frac{m_{2}}{2}\left(1+R_{2}\right) e^{-m_{2}|k|\left|\alpha-\alpha_{0}\right|}\right. \\
& -\frac{m_{1} T_{3}}{2 T_{2}}\left(1+R_{1}\right) e^{-m_{1}|k|\left(\alpha+\alpha_{0}\right)}-\frac{m_{2} T_{3}}{2 T_{2}}\left(1+R_{2}\right) e^{-m_{2}|k|\left(\alpha+\alpha_{0}\right)} \\
& +\frac{m_{1} T_{4}}{T_{2}}\left(1+R_{1}\right) e^{-|k|\left(m_{1} \alpha+m_{2} \alpha_{0}\right)} \\
& \left.+\frac{m_{2} T_{5}}{T_{2}}\left(1+R_{2}\right) e^{-|k|\left(m_{2} \alpha+m_{1} \alpha_{0}\right)}\right],
\end{aligned}
$$

where the upper sign is for the region $\alpha<\alpha_{0}$ and lower sign is for the region $\alpha>\alpha_{0}$, and $\alpha_{0}=H / h$,

$$
\begin{aligned}
& R_{1}=\frac{1+c}{a m_{1}^{2}-1}, R_{2}=\frac{1+c}{a m_{2}^{2}-1}, T_{1}=\frac{a R_{1}\left(m_{2}^{2}-m_{1}^{2}\right)}{a m_{2}^{2}-1}, \\
& T_{2}=\left(a m_{1} m_{2}+c\right)\left(m_{1} R_{1}-m_{2} R_{2}\right)+\left(m_{1}-m_{2}\right) a m_{1} m_{2} R_{1} R_{2}+\left(m_{1}-m_{2}\right) c, \\
& T_{3}=\left(a m_{1} m_{2}-c\right)\left(m_{1} R_{1}+m_{2} R_{2}\right)+\left(m_{1}+m_{2}\right) a m_{1} m_{2} R_{1} R_{2}-\left(m_{1}+m_{2}\right) c,
\end{aligned}
$$




$$
\begin{aligned}
& T_{4}=\left(a m_{2}^{2}-c\right) m_{2} R_{2}+a m_{2}^{3} R_{2}^{2}-m_{2} c, \\
& T_{5}=\left(a m_{1}^{2}-c\right) m_{1} R_{1}+a m_{1}^{3} R_{1}^{2}-m_{1} c .
\end{aligned}
$$

We note that quantities $R_{1}, R_{2}, T_{1}, T_{2}, T_{3}, T_{4}$ and $T_{5}$ are independent of $k$. Taking inversion and using appendix A, we get the following closed-form expressions for the displacements and stresses at any point of an orthotropic elastic half-space due to a normal line force acting at $(H, 0)$.

$$
\begin{aligned}
& U(\alpha, \beta)=\frac{P}{4 \pi T_{1}}\left[-m_{1} R_{1} \log \left\{\beta^{2}+m_{1}^{2}\left(\alpha-\alpha_{0}\right)^{2}\right\}\right. \\
& +m_{2} R_{2} \log \left\{\beta^{2}+m_{2}^{2}\left(\alpha-\alpha_{0}\right)^{2}\right\} \\
& -\frac{m_{1} R_{1} T_{3}}{T_{2}} \log \left\{\beta^{2}+m_{1}^{2}\left(\alpha+\alpha_{0}\right)^{2}\right\} \\
& -\frac{m_{2} R_{2} T_{3}}{T_{2}} \log \left\{\beta^{2}+m_{2}^{2}\left(\alpha+\alpha_{0}\right)^{2}\right\} \\
& +\frac{2 m_{1} R_{1} T_{4}}{T_{2}} \log \left\{\beta^{2}+\left(m_{1} \alpha+m_{2} \alpha_{0}\right)^{2}\right\} \\
& \left.+\frac{2 m_{2} R_{2} T_{5}}{T_{2}} \log \left\{\beta^{2}+\left(m_{2} \alpha+m_{1} \alpha_{0}\right)^{2}\right\}\right], \\
& V(\alpha, \beta)=\frac{P}{2 \pi T_{1}}\left[\mp \tan ^{-1}\left(\frac{\beta}{m_{1}\left|\alpha-\alpha_{0}\right|}\right) \pm \tan ^{-1}\left(\frac{\beta}{m_{2}\left|\alpha-\alpha_{0}\right|}\right)\right. \\
& +\frac{T_{3}}{T_{2}} \tan ^{-1}\left(\frac{\beta}{m_{1}\left(\alpha+\alpha_{0}\right)}\right)+\frac{T_{3}}{T_{2}} \tan ^{-1}\left(\frac{\beta}{m_{2}\left(\alpha+\alpha_{0}\right)}\right) \\
& \left.-\frac{2 T_{4}}{T_{2}} \tan ^{-1}\left(\frac{\beta}{\left|m_{1} \alpha+m_{2} \alpha_{0}\right|}\right)-\frac{2 T_{5}}{T_{2}} \tan ^{-1}\left(\frac{\beta}{m_{2} \alpha+m_{1} \alpha_{0}}\right)\right] \text {, } \\
& \sigma_{11}(\alpha, \beta)=\frac{P}{2 \pi T_{1}}\left[ \pm\left(a m_{1}^{2} R_{1}-c\right)\left\{\frac{m_{1}\left(\alpha_{0}-\alpha\right)}{\beta^{2}+m_{1}^{2}\left(\alpha_{0}-\alpha\right)^{2}}\right\}\right. \\
& \mp\left(a m_{2}^{2} R_{2}-c\right)\left\{\frac{m_{2}\left|\alpha-\alpha_{0}\right|}{\beta^{2}+m_{2}^{2}\left(\alpha-\alpha_{0}\right)^{2}}\right\} \\
& -\frac{T_{3}}{T_{2}}\left(a m_{1}^{2} R_{1}-c\right)\left\{\frac{m_{1}\left(\alpha+\alpha_{0}\right)}{\beta^{2}+m_{1}^{2}\left(\alpha+\alpha_{0}\right)^{2}}\right\} \\
& -\frac{T_{3}}{T_{2}}\left(a m_{2}^{2} R_{2}-c\right)\left\{\frac{m_{2}\left(\alpha+\alpha_{0}\right)}{\beta^{2}+m_{2}^{2}\left(\alpha+\alpha_{0}\right)^{2}}\right\} \\
& +\frac{2 T_{4}}{T_{2}}\left(a m_{1}^{2} R_{1}-c\right)\left\{\frac{\left|m_{1} \alpha+m_{2} \alpha_{0}\right|}{\beta^{2}+\left(m_{1} \alpha+m_{2} \alpha_{0}\right)^{2}}\right\} \\
& \left.+\frac{2 T_{5}}{T_{2}}\left(a m_{2}^{2} R_{2}-c\right)\left\{\frac{\left|m_{2} \alpha+m_{1} \alpha_{0}\right|}{\beta^{2}+\left(m_{2} \alpha+m_{1} \alpha_{0}\right)^{2}}\right\}\right] \text {, }
\end{aligned}
$$




$$
\begin{aligned}
\sigma_{12}(\alpha, \beta)= & \frac{P}{2 \pi T_{1}}\left[-\frac{m_{1}\left(1+R_{1}\right) \beta}{\beta^{2}+m_{1}^{2}\left(\alpha-\alpha_{0}\right)^{2}}+\frac{m_{2}\left(1+R_{2}\right) \beta}{\beta^{2}+m_{2}^{2}\left(\alpha-\alpha_{0}\right)^{2}}\right. \\
& -\frac{m_{1} T_{3}\left(1+R_{1}\right) \beta}{T_{2}\left\{\beta^{2}+m_{1}^{2}\left(\alpha+\alpha_{0}\right)^{2}\right\}}-\frac{m_{2} T_{3}\left(1+R_{2}\right) \beta}{T_{2}\left\{\beta^{2}+m_{2}^{2}\left(\alpha+\alpha_{0}\right)^{2}\right\}} \\
& \left.+\frac{2 m_{1} T_{4}\left(1+R_{1}\right) \beta}{T_{2}\left\{\beta^{2}+\left(m_{1} \alpha+m_{2} \alpha_{0}\right)^{2}\right\}}+\frac{2 m_{2} T_{5}\left(1+R_{2}\right) \beta}{T_{2}\left\{\beta^{2}+\left(m_{2} \alpha+m_{1} \alpha_{0}\right)^{2}\right\}}\right] .
\end{aligned}
$$

4.2 Deformation of a transversely isotropic elastic half-space

Following the same procedure, as adopted for an orthotropic case, we obtain the following closed-form expressions for displacements at any point of a transversely isotropic elastic half-space due to a normal line-load acting at $(H, 0)$.

$$
\begin{aligned}
U(\alpha, \beta)= & \frac{P}{4 \pi}\left[\frac{a-1}{a}\left\{\frac{\left(\alpha_{0}-\alpha\right)^{2}}{\beta^{2}+\left(\alpha_{0}-\alpha\right)^{2}}\right\}-\left(\frac{a+1}{2 a}\right) \log \left\{\beta^{2}+\left(\alpha_{0}-\alpha\right)^{2}\right\}\right. \\
& +\left(\frac{a+1}{a}\right)\left\{\frac{\left(\alpha_{0}+\alpha\right)^{2}}{\beta^{2}+\left(\alpha_{0}+\alpha\right)^{2}}\right\}-\frac{1}{2 a}\left(\frac{a^{2}+1}{a-1}\right) \log \left\{\beta^{2}+\left(\alpha_{0}+\alpha\right)^{2}\right\} \\
& \left.+2\left(\frac{a-1}{a}\right) \alpha_{0} \alpha\left\{\frac{\left(\alpha+\alpha_{0}\right)^{2}-\beta^{2}}{\left(\left(\alpha+\alpha_{0}\right)^{2}+\beta^{2}\right)^{2}}\right\}\right], \\
V(\alpha, \beta)= & \frac{P}{4 \pi}\left[-\left(\frac{a-1}{a}\right)\left(\alpha_{0}-\alpha\right)\left\{\frac{\beta}{\beta^{2}+\left(\alpha_{0}-\alpha\right)^{2}}\right\}\right. \\
& -\left(\frac{a+1}{a}\right)\left|\alpha_{0}-\alpha\right|\left\{\frac{\beta}{\beta^{2}+\left(\alpha_{0}+\alpha\right)^{2}}\right\}-\left(\frac{2}{a-1}\right) \tan ^{-1}\left(\frac{\beta}{\alpha+\alpha_{0}}\right) \\
& \left.+4 \alpha \alpha_{0}\left(\frac{a-1}{a}\right)\left\{\frac{\beta\left(\alpha+\alpha_{0}\right)}{\left(\beta^{2}+\left(\alpha_{0}+\alpha\right)^{2}\right)^{2}}\right\}\right] .
\end{aligned}
$$

\subsection{Deformation of an isotropic elastic half-space}

For an isotropic case $a=1 /\left(1-\alpha^{*}\right)$, using this in (87), we obtain the following closedform expressions for the displacements at any point of an isotropic elastic half-space due to a normal line-load acting at $(H, 0)$.

$$
\begin{aligned}
U(\alpha, \beta)= & \frac{P}{4 \pi}\left[\frac{\alpha^{*}\left(\alpha_{0}-\alpha\right)^{2}}{\beta^{2}+\left(\alpha_{0}-\alpha\right)^{2}}-\frac{\left(2-\alpha^{*}\right)}{2} \log \left\{\beta^{2}+\left(\alpha_{0}-\alpha\right)^{2}\right\}\right. \\
& +\frac{\left(2-\alpha^{*}\right)\left(\alpha_{0}+\alpha\right)^{2}}{\beta^{2}+\left(\alpha_{0}+\alpha\right)^{2}}-\frac{1}{2}\left(\frac{2-2 \alpha^{*}+\alpha^{* 2}}{\alpha^{*}}\right) \log \left\{\beta^{2}+\left(\alpha_{0}+\alpha\right)^{2}\right) \\
& \left.+2 \alpha^{*} \alpha_{0} \alpha\left\{\frac{\left(\alpha+\alpha_{0}\right)^{2}-\beta^{2}}{\left(\left(\alpha+\alpha_{0}\right)^{2}+\beta^{2}\right)^{2}}\right\}\right], \\
V(\alpha, \beta)= & \frac{P}{4 \pi}\left[\frac{-\alpha^{*} \beta\left(\alpha_{0}-\alpha\right)}{\beta^{2}+\left(\alpha_{0}-\alpha\right)^{2}}-\frac{\left(2-\alpha^{*}\right)\left(\alpha_{0}-\alpha\right) \beta}{\beta^{2}+\left(\alpha_{0}+\alpha\right)^{2}}\right. \\
& \left.-2\left(\frac{1-\alpha^{*}}{\alpha^{*}}\right) \tan ^{-1}\left(\frac{\beta}{\alpha+\alpha_{0}}\right)+\frac{4 \alpha_{0} \alpha \alpha^{*} \beta\left(\alpha+\alpha_{0}\right)}{\left\{\beta^{2}+\left(\alpha+\alpha_{0}\right)^{2}\right)^{2}}\right]
\end{aligned}
$$


The displacements for an isotropic elastic half-space obtained in (88) coincide with the corresponding results obtained by Maruyama (1966).

\section{Numerical results and discussion}

We wish to examine the effect of anisotropy of the elastic half-space. For numerical computations, we use the values of elastic constants given by Love (1944) for topaz material (orthotropic), which are

$$
a=2.15789, \quad b=2.67669, \quad c=0.96240 .
$$

To examine the effect of anisotropy of elastic medium, various distribution curves in figures 2 to 5 are plotted for orthotropic, transversely isotropic and isotropic elastic medium. For a transversely isotropic medium, the following relations

$$
b=a, \quad c=a-2
$$

are taken into consideration. For an isotropic elastic medium we consider $\alpha^{*}=0.6624$. In these figures, the normal and tangential dimensionless displacements at the surface level $\alpha=0$ and subsurface level $\alpha=0.5$ have been shown for $\alpha_{0}=1.0$. Figures 2 and 4 show the variation of dimensionless normal displacement with dimensionless horizontal distance for orthotropic, transversely isotropic and isotropic elastic medium. In each case it is found that as we move away from the point of application, the difference between the dimensionless displacements corresponding to orthotropic, transversely and isotropic elastic medium increases. Also it is found that in magnitude the difference between the normal displacements corresponding to

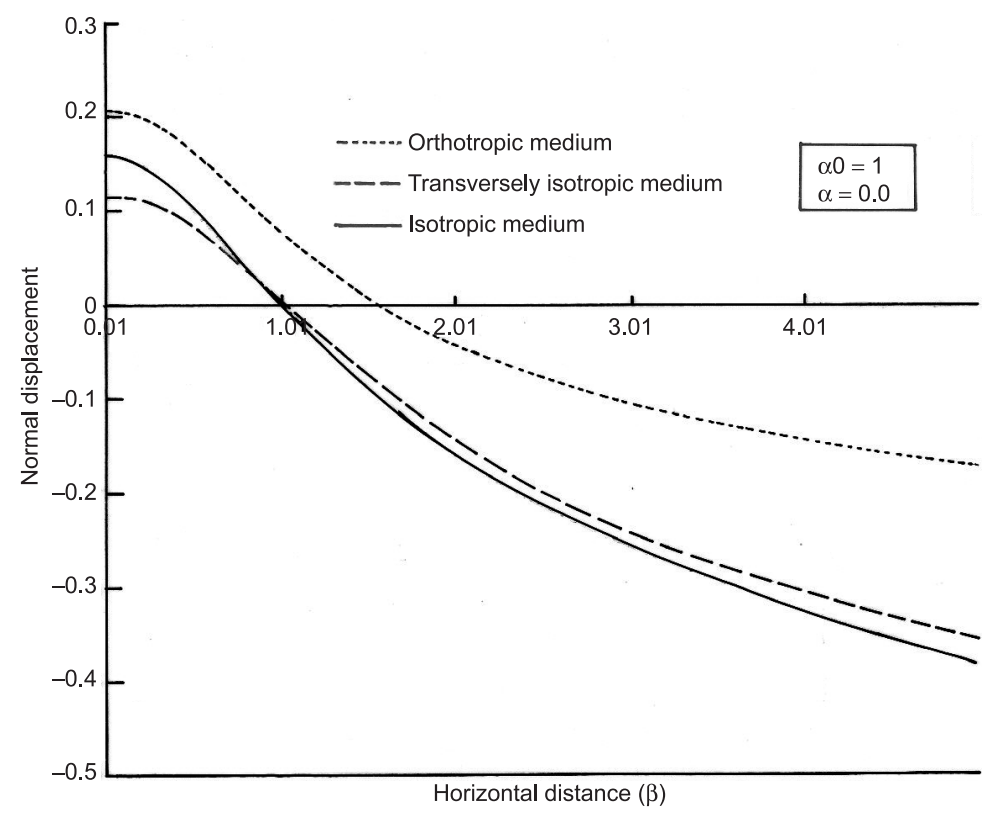

Figure 2. Variation of dimensionless normal displacement for orthotropic, transversely isotropic and isotropic elastic media. 


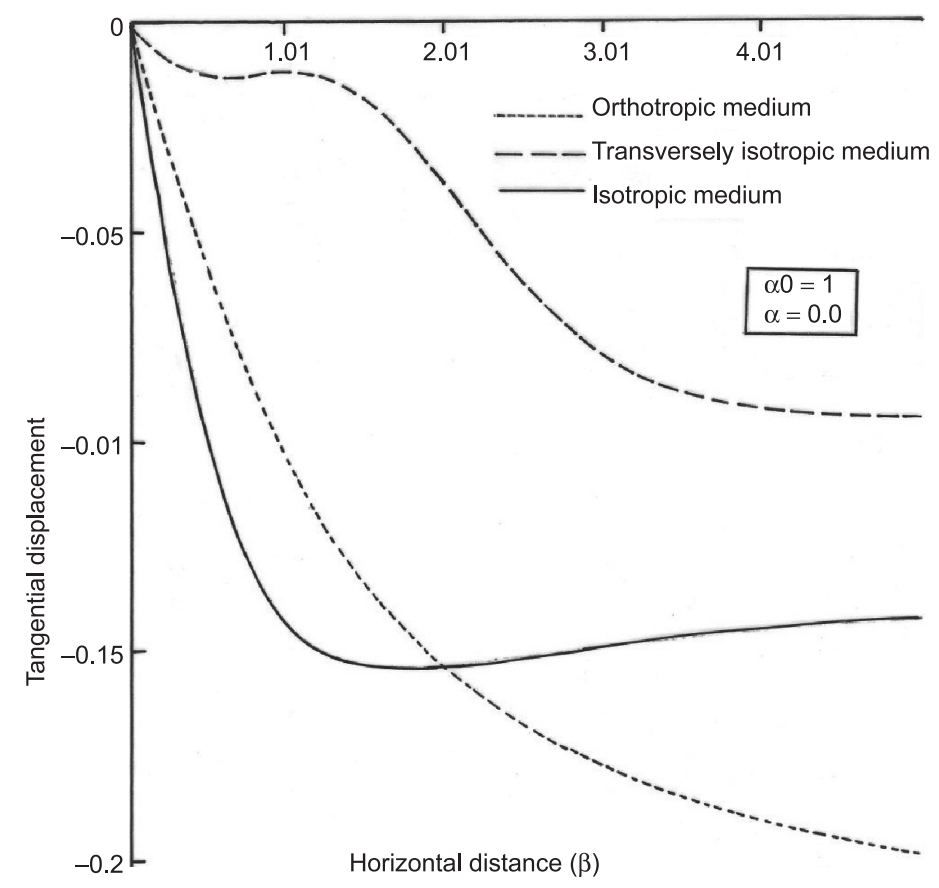

Figure 3. Variation of dimensionless tangential displacement for orthotropic, transversely isotropic and isotropic elastic media.

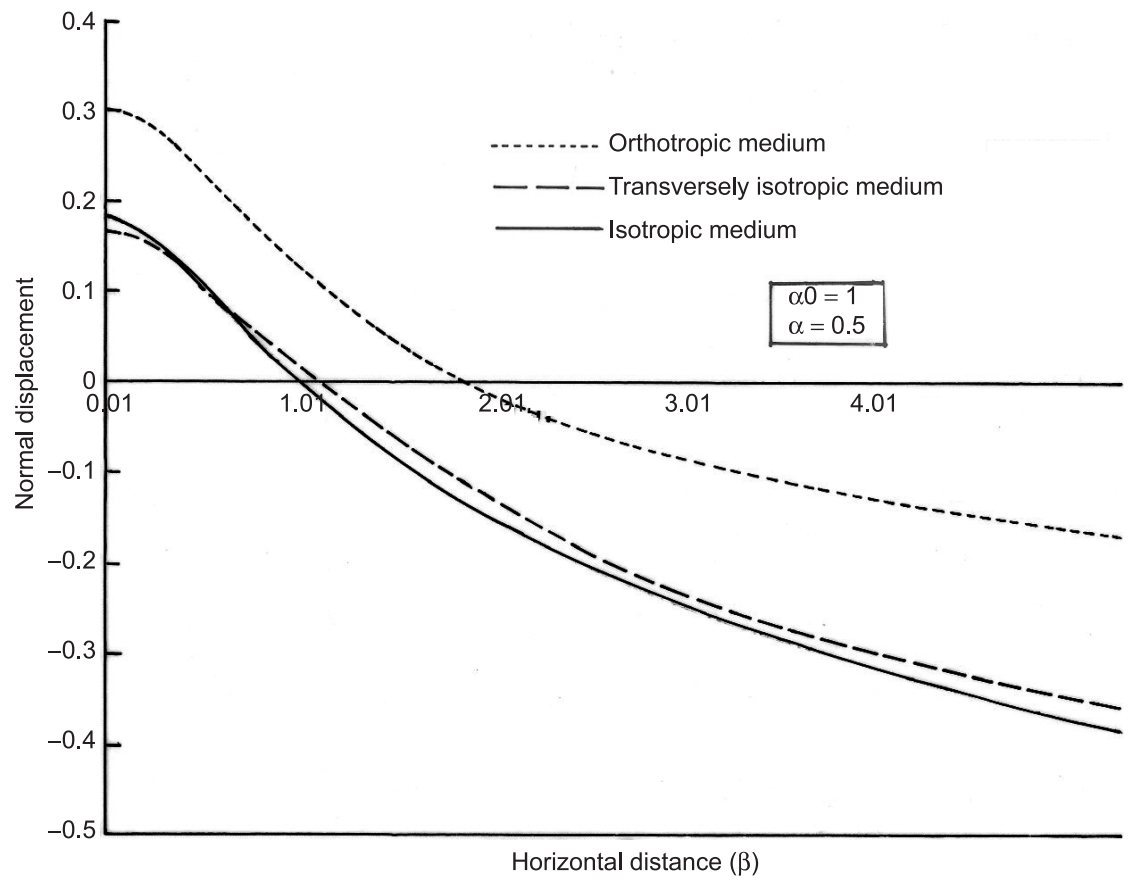

Figure 4. Variation of dimensionless normal displacement for orthotropic, transversely isotropic and isotropic elastic media. 


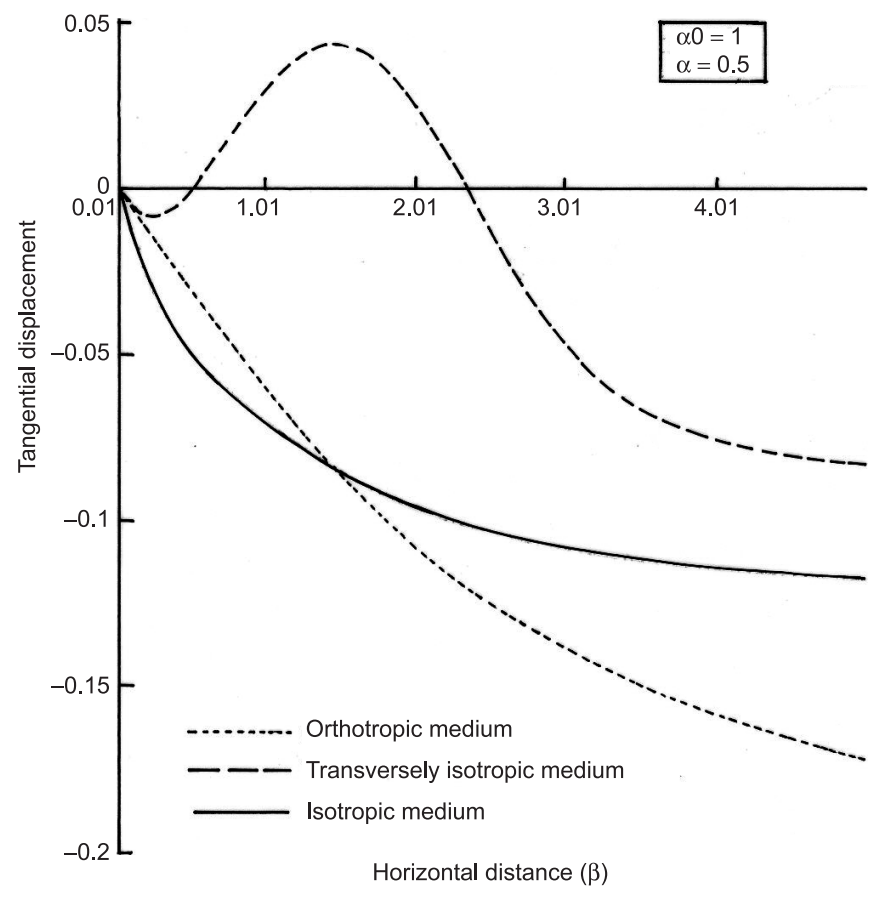

Figure 5. Variation of dimensionless tangential displacement distribution for orthotropic, transversely isotropic and isotropic elastic media.

orthotropic and isotropic is greater than that of the difference between transversely isotropic and isotropic. Figures 3 and 5 show the variation of dimensionless tangential displacement with dimensionless horizontal distance for orthotropic, transversely isotropic and isotropic elastic medium. As we move away from the point of application the difference between the dimensionless tangential displacement corresponding to orthotropic and isotropic elastic medium increases. While the difference between the dimensionless tangential displacements corresponding to isotropic and transversely isotropic medium decreases.

From these figures, it is concluded that the anisotropy is affecting the deformation substantially.

KS is grateful to the University Grants Commission, New Delhi for financial support.

Appendix $(x>0) \mathbf{A}$

(1) $\int_{-\infty}^{\infty} e^{-\imath k y} d k=2 \pi \delta(y)$

(2) $\int_{\infty}^{\infty}(|k|)^{-1} e^{-|k| x} e^{-l k y} d k=-\log \left(y^{2}+x^{2}\right)$ 
(3) $\int_{-\infty}^{\infty} k^{-1} e^{-|k| x} e^{-\iota k y} d k=-2 \iota \tan ^{-1}\left(\frac{y}{x}\right)$

(4) $\int_{-\infty}^{\infty} e^{-|k| x} e^{-\imath k y} d k=\frac{2 z}{y^{2}+z^{2}}$

(5) $\int_{-\infty}^{\infty} \frac{k}{|k|} e^{-|k| x} e^{-\imath k y} d k=\frac{-2 \iota y}{y^{2}+x^{2}}$

(6) $\int_{-\infty}^{\infty} k e^{-|k| x} e^{-\imath k y} d k=\frac{-4 \iota y x}{\left(y^{2}+x^{2}\right)^{2}}$

(7) $\int_{-\infty}^{\infty}|k| e^{-|k| x} e^{-\imath k y} d k=\frac{2\left(z^{2}-y^{2}\right)}{\left(z^{2}+y^{2}\right)^{2}}$

(8) $\int_{-\infty}^{\infty} k^{2} e^{-|k| z} e^{-\imath k y} d k=\frac{4 x\left(x^{2}-3 y^{2}\right)}{\left(x^{2}+y^{2}\right)^{3}}$

(9) $\int_{-\infty}^{\infty} k|k| e^{-|k| x} e^{-\imath k y} d k=\frac{-4 \iota y\left(3 z^{2}-y^{2}\right)}{\left(z^{2}+y^{2}\right)^{3}}$

\section{References}

Chou Y T 1976 On antiplane line force in a two-phase anisotropic medium. Phys. Stat. Sol. 34: 645650

Dziewonski A M, Anderson D L 1981 Preliminary reference Earth model. Phys. Earth Planet. Inter. 25: 297-356

Garg N R, Madan D K, Sharma R K 1996 Two-dimensional deformation of an orthotropic elastic medium due to seismic sources. Phys. Earth Planet. Inter. 94: 43-62

Garg N R, Kumar R, Goel A, Miglani A 2003 Plane strain deformation of an orthotropic elastic medium using an eigenvalue approach. Earth, Planets Space 55: 3-8

Maruyama T 1966 On two-dimensional elastic dislocations in an infinite and semi-infinite medium. Bull. Earthquake Res. Inst. 44: 811-871

Pan E 1989 Static response of a transversely isotropic and layered half-space to general dislocation sources. Phys. Earth Planet. Inter. 58: 103-117

Ross S L 1984 Differential equations 3rd edn (New York: John Wiley and Sons)

Small J C, Booker J R 1984 Finite layer analysis of layered elastic materials using a flexibility approach, Part I - strip loading. Int. J. Numer. Methods Engg. 21: 1025-1037

Ting T C T 1995 Antiplane deformation of anisotropic elastic materials. In Recent advances in elasticity, viscoelasticity and inelasticity: Series in advances in mathematics in applied sciences (ed.) K R Rajgopal (Singapore: World Scientific) 26: 150-179 